\title{
The influence of meridional ice transport on Europa's ocean stratification and heat content
}

Peiyun Zhu, ${ }^{1}$ Georgy E. Manucharyan, ${ }^{2}$ Andrew F. Thompson, ${ }^{2}$

\author{
Jason C. Goodman, ${ }^{3}$ and Steven D. Vance ${ }^{4}$
}

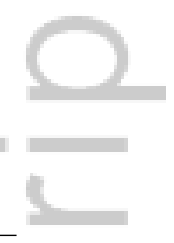

Corresponding author: Peiyun Zhu, Department of Earth and Environmental Sciences, University of Michigan, Ann Arbor, Michigan, USA. (zhpeiyun@umich.edu)

${ }^{1}$ Department of Earth and Environmental

Sciences, University of Michigan, Ann

Arbor, Michigan, USA.

${ }^{2}$ Department of Environmental Science

and Engineering, California Institute of

Technology, Pasadena, California, USA.

${ }^{3}$ Department of Physics, Wheaton

College, Norton, Massachusetts, USA

${ }^{4}$ Jet Propulsion Laboratory, California

Institute of Technology, Pasadena,

California, USA.

This is the author manuscript accepted for publication and has undergone full peer review but has not been through the copyediting, typesetting, pagination and proofreading process, which

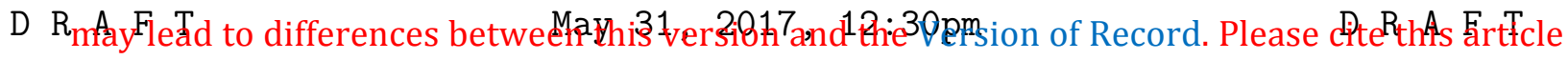
as doi: 10.1002/2017GL072996

This article is protected by copyright. All rights reserved. 
${ }_{4} \quad$ Jupiter's moon Europa likely hosts a saltwater ocean beneath its icy sur-

${ }_{5}$ face. Geothermal heating and rotating convection in the ocean may drive a

6 global overturning circulation that redistributes heat vertically and merid-

7 ionally, preferentially warming the ice shell at the equator. Here, we assess

s the previously unconstrained influence of ocean-ice coupling on Europa's ocean

9 stratification and heat transport. We demonstrate that a relatively fresh layer

${ }_{10}$ can form at the ice-ocean interface due to a meridional ice transport forced

${ }_{11}$ by the differential ice shell heating between the equator and the poles. We

${ }_{12}$ provide analytical and numerical solutions for the layer's characteristics, high-

${ }_{13}$ lighting their sensitivity to critical ocean parameters. For a weakly-turbulent

${ }_{14}$ and highly-saline ocean, a strong buoyancy gradient at the base of the fresh-

${ }_{15}$ water layer can suppress vertical tracer exchange with the deeper ocean. As

${ }_{16}$ a result, the freshwater layer permits relatively warm deep ocean temper-

${ }_{17}$ atures.

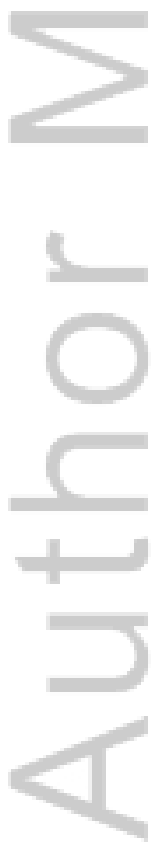

D R A F T

May $31,2017,12: 30 \mathrm{pm}$

D R A F T 


\section{Introduction}

Jupiter's moon Europa is one of multiple confirmed ocean worlds [Nimmo and Pap-

19 palardo, 2016]. Evidence for an extant subsurface ocean comes from measurements by the

${ }_{20}$ Galileo spacecraft indicating an induced response to the changing direction of Jupiter's

${ }_{21}$ magnetic field, consistent with the existence of an electrical conductor near the surface

${ }_{22}$ [Kivelson et al., 2000]. Based on gravity measurements, the rocky seafloor is $80-170 \mathrm{~km}$

${ }_{23}$ below the surface [Anderson et al., 1998]. The ocean is in communication with the surface

24 on time scales shorter than $100 \mathrm{Myr}$, as indicated by Europa's complex surface geology

${ }_{25}$ [e.g., Pappalardo et al., 1998] and sparsity of craters [Zahnle et al., 2008]. This interaction

${ }_{26}$ controls the flux of surface-derived oxidants into the ocean [Vance et al., 2016] and influ-

${ }_{27}$ ences the ocean's dynamics in ways that have not been thoroughly evaluated to date. The

${ }_{28}$ ocean's composition, stratification, and circulation influence chemical exchange, such that

${ }_{29}$ an understanding of Europa's dynamical properties could help to assess whether Europa

so can support life [e.g., Schulze-Makuch and Irwin, 2002; Irwin and Schulze-Makuch, 2003].

Geothermal heat from the seafloor and loss of heat through the ice shell are critical mechanisms driving Europa's ocean circulation. Buoyant plumes confined by Coriolis

33 forces may act to regionally transmit heat and materials directly between the seafloor ${ }_{34}$ and ice [Thomson and Delaney, 2001; Goodman et al., 2004; Vance and Goodman, 2009].

${ }_{35}$ However, larger-scale circulation features may develop through turbulent convection and ${ }_{36}$ through rotational constraints [Travis et al., 2012; Soderlund et al., 2014]. Critically, these ${ }_{37}$ prior studies have focused exclusively on the ocean and prescribed either a uniform surface ${ }_{38}$ temperature or a spatial distribution of surface heat fluxes. 
39 The pole-to-equator temperature variation on Europa ( 40 K) [Spencer et al., 1999;

${ }_{40}$ Rathbun et al., 2010] could support meridional variations in ice thickness that will also

${ }_{41}$ depend on the heat flux at the ocean-ice interface. The meridional ice thickness variations

${ }_{42}$ are estimated to be at most $3 \mathrm{~km}$, and zonal variations due to long-wavelength topography

43 less than $7 \mathrm{~km}$ [Nimmo et al., 2007]. Any variations in ice thickness will establish a pressure

${ }_{44}$ gradient, which can induce ice transport [Vance and Goodman, 2009]. This can occur by

${ }_{45}$ two mechanisms: the so-called ice pump [Lewis and Perkin, 1986], and down-thickness

46 gradient ice flow [Goodman and Pierrehumbert, 2003].

${ }_{47} \quad$ Over sufficiently long time scales, thicker ice at the poles implies continuous transport

${ }_{48}$ of ice equatorward. At the equator (poles), the addition (removal) of ice requires prefer-

${ }_{49}$ ential melting (growth) to maintain steady state conditions. In a weakly turbulent and

${ }_{50}$ saline ocean, freshwater fluxes at the equator can dilute the upper ocean to form a stable

${ }_{51}$ layer with lower salinity than the deep ocean, hence defined as a "freshwater" layer. In a ${ }_{52}$ dilute ocean [e.g., Zolotov and Shock, 2001; McKinnon and Zolensky, 2003] with buoyancy

${ }_{53}$ depending mainly on temperature, a freshwater lens can also be stable due to the nega-

${ }_{54}$ tive thermal expansion coefficient of water for hydrostatic pressures less than $\sim 25 \mathrm{MPa}$

${ }_{55}$ (Europa ice thickness less than $\sim 17 \mathrm{~km}$ ) [Melosh et al., 2004].

${ }_{56}$ The strength and turbulent properties of Europa's ocean circulation are uncertain. For

${ }_{57}$ example, Soderlund et al. [2014] demonstrates the possibility for an energetic convectively-

58 driven overturning ocean circulation that enhances the equatorial ocean heat fluxes. Other

${ }_{59}$ studies suggest alternative, less vigorous circulation regimes [e.g., Vance and Goodman,

${ }_{60}$ 2009; Jansen, 2016] with lower turbulent levels. However, these studies do not account 
${ }_{61}$ for freshwater fluxes associated with the freezing/melting of the ice. Thus, the existence

${ }_{62}$ of the salt stratification of Europa's ocean remains an open question.

Here we introduce a conceptual, two-column model to quantify the physical processes

${ }_{64}$ that may give rise to a freshwater layer beneath Europa's ice shell. Using this model,

${ }_{65}$ we explore the sensitivity of the layer to key ocean characteristics, including its average

${ }_{66}$ salinity, the strength of the upper ocean turbulence, and the equator-to-pole ocean heat

${ }_{67}$ flux. The presence of a freshwater layer under the ice can suppress the efficiency of heat

${ }_{68}$ exchange with the deep ocean due to a buoyancy contrast at the interface between the

${ }_{69}$ layer and the deep ocean. We explore under which conditions this layer can influence deep

70 ocean temperatures.

\section{Model description}

Our approach is to develop a minimal model that captures the essential dynamics lead-

72 ing to the formation of compositional stratification in low-latitude regions of Europa's

73 ocean. An extreme but still insightful truncation is to consider two vertical columns, one

74 at the equator (low latitudes) and one at the pole (high latitudes), to represent merid-

${ }_{75}$ ional gradients in ice thickness and ocean properties (Figure 1). An advantage of this

76 approach is the derivation of analytical scalings that indicate the sensitivity (e.g., power

77 law dependence) of the freshwater layer characteristics to Europa's properties.

\subsection{Ice thickness balance}

78 The global heat budget governs the distribution of ice shell thickness. In our model, ${ }_{79}$ the positive heat flux from the ocean into the ice is transferred vertically through the ice 
so by thermal diffusion. The temperature at the ocean-ice interface is fixed at the freezing

${ }_{81}$ point $T_{f}$, which may vary with pressure and salinity.

The ocean-ice heat flux $F_{\text {ocn }}$ at the equator and the poles may be different, reflected

${ }_{83}$ in a parameter $\Delta F_{\mathrm{ocn}}=F_{\mathrm{ocn}}^{e}-F_{\mathrm{ocn}}^{p}$; throughout this paper superscripts $e$ and $p$ denote

${ }_{84}$ variables of the equator and the pole columns, respectively. In studies by [e.g., Goodman

${ }_{85}$ et al., 2004; Jansen, 2016], the ice is considered to be in a steady state governed by a

${ }_{86}$ one-dimensional vertical balance. However, positive lateral gradients in ice thickness will

${ }_{87}$ induce an equatorward ice or freshwater transport $F_{h}\left(\mathrm{~m} \mathrm{~s}^{-1}\right)$ that results in ice formation

${ }_{88}$ at high latitudes and freshwater accumulation at low latitudes. Two physical mechanisms

${ }_{89}$ give rise to $F_{h}$ : (i) down-gradient thickness transport [Goodman and Pierrehumbert, 2003]

so and (ii) the ice pump, which arises from the dependence of $T_{f}$ on pressure (ice thickness)

${ }_{91}$ and composition [Lewis and Perkin, 1986]. By introducing $F_{h}$, we couple the ice dynamics

${ }_{92}$ to the ocean and are able to quantitatively explore their interactions.

The ice thickness balance is governed by

$$
\begin{aligned}
L \frac{d h^{e}}{d t} & =\frac{\kappa_{\mathrm{ice}}\left(T_{f}-T_{s}^{e}\right)}{h_{0}^{e}}+L F_{h}-\left(F_{\mathrm{ocn}}+\Delta F_{\mathrm{ocn}}\right), \\
L \frac{d h^{p}}{d t} & =\frac{\kappa_{\mathrm{ice}}\left(T_{f}-T_{s}^{p}\right)}{h_{0}^{e}+\Delta h}-L F_{h}-F_{\mathrm{ocn}},
\end{aligned}
$$

${ }_{94}$ where $h^{e}$ and $T_{s}^{e}\left(h^{p}\right.$ and $\left.T_{s}^{p}\right)$ are the instantaneous ice thickness and surface temperature

${ }_{95}$ at low (high) latitudes, $\kappa_{\text {ice }}$ is the thermal conductivity of ice, $L$ is the latent heat of ice

${ }_{96}$ fusion (Table 1), $h_{0}^{e}$ is the equilibrium ice thickness at the equator and $\Delta h=h^{p}-h^{e}>0$

${ }_{97}$ is the pole-to-equator difference in the ice thickness. From left to right, the terms on the

${ }_{98}$ right hand side of equations (1) and (2) represent the heat loss due to diffusion through

99 the ice, the thickness flux caused by ice transport, and the ocean-ice heat flux. 
Relative variations in Europa's ice thickness $\left[\Delta h / h^{e} \sim 20 \%\right.$; Nimmo et al., 2007] are much smaller than the variation of surface temperature $\left(\Delta T / T^{e} \sim 110 \%\right.$, Table 1$)$. Thus we can simplify the ice thickness equations by ignoring $\Delta h$ in (2). The steady state thickness flux can then be estimated from (1) and (2) as:

$$
F_{h}=\frac{\kappa_{\text {ice }}\left(T_{s}^{e}-T_{s}^{p}\right)}{2 h_{0} L}+\frac{\Delta F_{\text {ocn }}}{2 L} .
$$

where $S_{0}$ is the average salinity of Europa's ocean, $\rho_{i}$ and $\rho$ are densities of ice and water, respectively (Table 1). Next, we examine the depth of the freshwater layer, which depends on the ocean's circulation.

\subsection{Salt balance in a freshwater layer}

We simplify the meridional distribution of the freshwater layer by considering a layer with depth $d$ in the low-latitude column, and no freshwater layer in the high-latitude column. Thus, the ocean is partitioned into three regions or boxes (Figure 1), overlaid by the ice shell. The freshwater layer is represented by the upper equatorial box, with 
${ }_{112}$ salinity $S^{e}$. We assume a uniform salinity $S_{0}$ for the rest of the ocean, which implies a 114 of salt for the ocean.

To balance the melting at low latitudes due to the equatorward ice transport, ice forms (and rejects brine) at high latitudes. This is equivalent to a lateral salt flux out of the freshwater layer $\left(F_{s}\right.$ in Figure 1). Additionally, turbulent salt and heat transport may occur across the interface between the layer and the deep ocean in response to the vertical velocity shear of a mean-flow circulation, as suggested by Soderlund et al. [2014]. In a steady state, $F_{s}$ is balanced by turbulent mixing and diffusion of salt from the deep ocean. This balance can be written in the following way:

$$
\left(c u^{*}+\frac{\kappa}{d}\right) \Delta S=\left(S_{0}-\Delta S\right) \frac{\rho_{i}}{\rho} F_{h},
$$

115
May $31,2017,12: 30 \mathrm{pm}$

$$
c=1.5 R i^{-3 / 2}, \quad R i=\frac{d g \beta \Delta S}{u^{* 2},}, \quad \Delta S=S_{0}-S^{e} .
$$

The Richardson number defines the ratio of the vertical stratification (reflected by a salinity contrast $\Delta S$ ) to the vertical velocity shear, and indicates (for $R i \gg 1$ ) the stratifica- 
${ }_{121}$ tion's ability to suppress turbulent mixing (Eq. 6). Vertical heat transport at the ice-ocean

${ }_{122}$ interface at low latitudes is parameterized in the same way, i.e. $F_{\mathrm{ocn}}^{e}=\rho C_{P} c_{\text {ice }} u^{*}\left(T_{e}-T_{f}\right)$,

${ }_{123}$ where $c_{\text {ice }}$ is the entrainment rate at the ice-ocean interface at low latitudes and has a

${ }_{124}$ fixed value.

Since the freshwater layer is in direct contact with the ice, its near-freezing temperature and low salinity have opposing effects on buoyancy. The relative importance of salinity and temperature is expressed through the ratio $\alpha \Delta T / \beta \Delta S$, where $\beta$ and $\alpha$ are the saline and thermal expansion coefficients, respectively (Table 1). When this ratio is small, we

129 can approximate the buoyancy contrast as $\Delta b=g \beta \Delta S$, which yields the relationship ${ }_{130}$ for $R i$ in (6). Combining the definition of $\Delta S$ in (6) with (5),,$R i$, and $\Delta S$ can be ${ }_{131}$ determined as functions of average salinity $S_{0}$, freshwater layer depth $d$ and the turbulent ${ }_{132}$ velocity $u^{*}$. Below, we explore the parameter regimes under which the freshwater layer ${ }_{133}$ can affect the stratification and heat content of Europa's ocean.

\section{Results}

\subsection{Meridional thickness flux of ice}

We assume that in steady state, the polar ocean-ice heat flux is equivalent to the geothermal heat flux at the seafloor (i.e. $F_{\mathrm{ocn}}^{p}=F_{b}$ ). A range of $F_{b}$ has been applied ${ }_{136}$ in studies of Europa's ocean (Section 4; Table 1). Here, we adopt a reference value of $F_{b}=0.01 \mathrm{~W} \mathrm{~m}{ }^{-2}$. The thickness flux $F_{h}$ depends on equator-to-pole differences in the ice surface temperature and the heat flux at the ocean-ice interface (Eq. 3). If we assume ${ }_{139}$ the equatorial heat flux to be $40 \%$ larger than at the poles (i.e. $\Delta F_{\text {ocn }} / F_{\text {ocn }}^{p}=0.4$ ) as in Soderlund et al. [2014], then the two terms in Eq. 3 contribute comparably to 
${ }_{141} F_{h}, O\left(10^{-11}\right) \mathrm{m} \mathrm{s}^{-1}$, or $\sim 7 \times 10^{-4} \mathrm{~m} \mathrm{yr}^{-1}$. Note that a strong ocean-ice heat flux 142 at the poles $\left(\Delta F_{\text {ocn }}<0\right)$ can overcome the positive surface temperature term, and the ${ }_{143}$ thickness transport can become poleward $\left(F_{h}<0\right)$, leading to freshwater formation at ${ }_{144}$ high latitudes. The mathematical descriptions for the freshwater layer located either at

\subsection{Critical ranges of the freshwater layer depth}


Combining (5) and (6), and using the assumption that $c u^{*} \gg \kappa / d$ we obtain

$$
d_{\min }=\frac{0.84 u^{* 8 / 3}}{\left(\frac{\rho_{i}}{\rho} F_{h}\right)^{2 / 3} g \beta S_{0}} .
$$

Details of the derivation are available in the supporting information. Given the second criterion, $c<c_{\text {ice }}$, using the definitions for $c(6)$ and $c_{\text {ice }}=10^{-3}$, and assuming $S_{0} \gg \Delta S$ results in

$$
d_{\max }=\frac{0.13 u^{* 3}}{\frac{\rho_{i}}{\rho} F_{h} g \beta S_{0}} .
$$


${ }_{178}$ properties (Table 1). Freshwater characteristics for a $\mathrm{MgSO}_{4}$ ocean are provided in the ${ }_{180}$ case above varies from $10^{-4}$ to $0.2 \mathrm{psu}$ (Figure 2 ).

\subsection{Temperature contrast and minimum average salinity}

Ocean heat content depends not only on the geothermal heat flux but also on the efficiency of the heat exchange with the ice. The freshwater layer functions as a blanket that partially insulates the deep ocean from the ice, and may create a stronger vertical temperature gradient than an ocean without the layer. To quantify this insulating effect, we consider the heat budget of the deep ocean for which heat transport into the freshwater layer balances geothermal heating:

$$
\frac{F_{b}}{\rho C_{P}}=\left(c u^{*}+\frac{\kappa}{d}\right) \Delta T
$$

$$
\Delta T=\frac{F_{b} \Delta S}{C_{P} \rho_{i} F_{h} S_{0}}=\frac{2.25 \rho^{2} F_{b} u^{* 8}}{C_{P}\left(d g \beta \rho_{i} F_{h} S_{0}\right)^{3}} .
$$

The real dependence of $\Delta T$ on $u^{*}$ is obscured here because of the additional dependence of $d$ on $u^{*}$. However, using (7) and (8), $\Delta T$ is independent of $u^{*}$ for $d=d_{\min }$ and $\Delta T \sim u^{*-1}$ 
189 for $d=d_{\max }$. This is consistent with a weakening of $\Delta T$ in response to stronger mixing.

Accounting for the uncertainty of geothermal heat flux $F_{b}$ (Table 1), the range of minimum $S_{0}$ is $28-200 \mathrm{psu}$ and $16-100 \mathrm{psu}$ for magnesium sulfate and seawater, respectively. This range of salinities is plausible; maximum salinities inferred from the induced magnetic field's amplitude are 200 psu for magnesium sulfate [Hand and Chyba, 2007] and 100 psu for seawater [Schilling et al., 2007]. Note that the minimum salinity requirement also varies with $\Delta F_{\text {ocn }}$ through its dependence on $F_{h}$ (Eq. 3).

\subsection{Sensitivity to $S_{0}, F_{b}$ and $F_{h}$}

Here, we examine the sensitivity of the freshwater-induced stratification to $S_{0}, F_{b}$, and $F_{h}$, whose values vary within the ranges suggested by previous studies (Table 1 ). When the deep ocean is saltier, the freshwater layer tends to be thinner, i.e., $d_{\min }$ and $d_{\max }$ decrease with $S_{0}$ (Eq. 7 and 8). This is because $R i$ is proportional to both $d$ and $\Delta S$; a smaller $d$ requires a larger $\Delta S$ to achieve the same mixing conditions (the same $R i$ value). 
can cause differential freezing of ice at the poles and melting at the equator, creating a freshwater flux at the top of the ocean. Over sufficiently long time scales, a persistent freshwater flux can form a diluted upper ocean layer, or a "freshwater" layer under the ice shell at low latitudes. Density stratification at the base of the layer affects the turbulent exchange of heat and salt with the deep ocean. Under a wide range of parameters, the layer acts as a blanket that partially isolates the deep ocean from the ice shell, allowing it to efficiently accumulate heat from below. As a result, deep ocean temperatures can exceed the expected adiabat by $4 \times 10^{-4} \mathrm{~K}$ to $0.6 \mathrm{~K}$, depending on both the bulk characteristics of the layer and the turbulent properties of the ocean. As predicted by our model, the energetic circulation proposed by Soderlund et al. [2014] would prohibit the formation of a freshwater layer. However, other circulation regimes with weaker turbulence [e.g., Vance and Goodman, 2009; Jansen, 2016] could support a freshwater layer in Europa's ocean.

We describe both analytical and numerical solutions for the depth of the freshwater layer, and for the magnitude of the vertical temperature and salinity contrasts. The critical depth range for freshwater layer formation is mainly controlled by the strength of upper ocean turbulence and is sensitive to the average salinity of Europa's ocean. With stronger turbulence and lower average salinity, the freshwater layer tends to extend deeper. A process that is not addressed in this model is the spreading of the freshwater layer to higher latitudes to counteract the lateral density gradient. The omission of this effect implies that freshwater layer depths calculated in this study are upper bounds.

The aim of the present conceptual model is to highlight key processes that can affect the heat and salt balances of the ocean. The model uses basic parameterizations of various 
${ }_{248}$ physical processes, so it is important to note where its assumptions may lead to unphysical

results. First, our model adopts a shear-driven parameterization of stratified turbulence.

Because there are no observations of any properties of upper ocean turbulence in Europa,

we devote further attention to different representations of the turbulent exchange at the

layer interfaces in the supporting information [Baines, 1975; Shrinivas and Hunt, 2014;

Kumagai, 1984] to demonstrate the similarity of our turbulent parameterization to that of vertical plume-driven turbulence. Our conclusions are not sensitive to the choice of turbulent parameterization as long as the adopted parameterization causes stratification to suppress the efficiency of turbulent transport. Second, we neglect meridional heat transport via global overturning circulation or by ocean eddies, which can modify the differential ocean heat flux at the base of the ice shell $\left(\Delta F_{\text {ocn }}\right)$. These effects must be included to construct a fully-coupled system for Europa's ice and ocean. This feedback cannot be determined at present due to the uncertainty in the nature of the circulation and heat transport processes in Europa's ocean. Nevertheless, the effects of lateral heat transport or other factors that influence $F_{h}$ through $\Delta F_{\text {ocn }}$ (e.g., ice convection, tidal heating, and freezing point variations at the ice-ocean interface) can be determined from the sensitivity of the vertical stratification to $\Delta F_{\text {ocn }}$ (Section 3.4). With the above caveats in mind, our model exhibits a broad parameter space under which a freshwater layer can exist. While some of those parameters are mutually dependent, our results are cause for

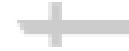

further investigation of Europa's upper ocean stratification due to the global exchange of heat between Europas ocean and ice. 
284 sharing issues

\section{References}

Observations from NASA'S planned Europa Clipper Mission [Pappalardo et al., 2016] and ESA's planned Jupiter ICy satellite Explorer mission [Grasset et al., 2013], will contribute to determining whether a freshwater layer exists, in particular by constraining the surface temperature distribution, the salinity of Europa's ocean and variations in its ice thickness. Such findings may in turn offer insight into Europa's habitability by helping to constrain the fluxes of energy and potential nutrients between the ice and ocean.

Acknowledgments. PZ completed this work through Caltech's Summer Undergraduate Research Fellowship (SURF) program; we thank the organizers for their support. GEM was supported by the Stanback Postdoctoral Fellowship Fund at Caltech. This work was partially supported by Strategic Research and Technology funds from the Jet Propulsion Laboratory, Caltech, and by the Icy Worlds node of NASA's Astrobiology Institute (13-13NAI7_2-0024). The research was carried out at the Jet Propulsion Laboratory, California Institute of Technology, under a contract with the National Aeronautics and Space Administration. The numerical model based on equations described in this paper produced figures that include all of the numerical information, so there are no data

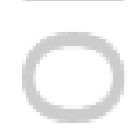

Anderson, J., G. Schubert, R. Jacobson, E. Lau, W. Moore, and W. Sjogren (1998), Europa's differentiated internal structure: inferences from four Galileo encounters, Science, $281(5385), 2019-2022$. 
Baines, W. (1975), Entrainment by a plume or jet at a density interface, J. Fluid Mech., 68(1), 309-320.

Goodman, J. C., and R. T. Pierrehumbert (2003), Glacial flow of floating marine ice in "snowball earth", Journal of Geophysical Research-Oceans, 108(C10), 3308.

Goodman, J. C., G. C. Collins, J. Marshall, and R. T. Pierrehumbert (2004), Hydrothermal plume dynamics on Europa: Implications for chaos formation, Journal of Geophysical Research-Planets, 109 (E3), E03,008, doi:10.1029/2003JE002,073.

Grasset, O., M. Dougherty, A. Coustenis, E. Bunce, C. Erd, D. Titov, M. Blanc, A. Coates, P. Drossart, L. Fletcher, H. Hussmann, R. Jaumann, N. Krupp, J.-P. Lebreton, O. Prieto-Ballesteros, P. Tortora, F. Tosi, and T. V. Hoolst (2013), JUpiter ICy moons Explorer (JUICE): An $\{\mathrm{ESA}\}$ mission to orbit Ganymede and to characterise the Jupiter system, Planetary and Space Science, 78(0), 1 - 21, doi:10.1016/j.pss.2012.12.002.

Hand, K., and C. Chyba (2007), Empirical constraints on the salinity of the europan ocean and implications for a thin ice shell, Icarus, 189(2), 424-438.

Irwin, L. N., and D. Schulze-Makuch (2003), Strategy for modeling putative multilevel ecosystems on Europa, Astrobiology, 3(4), 813-821.

Jansen, M. F. (2016), The turbulent circulation of a snowball earth ocean, Journal of Physical Oceanography, 46(6), 1917-1933.

Jenkins, A. (1991), A one-dimensional model of ice shelf-ocean interaction, Journal of Geophysical Research, 96(C11), 20,671-20,677.

Kit, E., E. Berent, and M. Vajda (1980), Vertical mixing induced by wind anda rotating screen in a stratified fluidin a channel, Journal of Hydraulic Research, 18(1), 35-58. 
Kivelson, M., K. Khurana, C. Russell, M. Volwerk, R. Walker, and C. Zimmer (2000), Galileo magnetometer measurements: A stronger case for a subsurface ocean at Europa, Science, 289, 1340-1343.

Kumagai, M. (1984), Turbulent buoyant convection from a source in a confined two-layered region, J. Fluid Mech., 147(1), 105-131.

Lewis, and Perkin (1986), Ice pumps and their rates, Journal of Geophysical Research, $91,11,756-11,762$.

Lowell, R. P., and M. DuBose (2005), Hydrothermal systems on Europa, Geophysical Research Letters, 32(5), L05,202, doi:10.1029/2005GL022,375.

Manucharyan, G. E., and C. Caulfield (2015), Entrainment and mixed layer dynamics of a surface-stress-driven stratified fluid, Journal of Fluid Mechanics, 765, 653-667.

McDougall, T. J., and P. M. Barker (2011), Getting started with TEOS-10 and the Gibbs Seawater (GSW) oceanographic toolbox, SCOR/IAPSO WG, 12\%, 1-28.

McKinnon, W. B., and M. E. Zolensky (2003), Sulfate content of Europa's ocean and shell: Evolutionary considerations and some geological and astrobiological implications, Astrobiology, 3(4), 879-897.

McPhee, M. G., C. Kottmeier, and J. H. Morison (1999), Ocean heat flux in the central Weddell Sea during winter, Journal of Physical Oceanography, 29(6), 1166-1179.

Melosh, H. J., A. G. Ekholm, A. P. Showman, and R. D. Lorenz (2004), The temperature of Europa's subsurface water ocean, Icarus, 168(2), 498-502.

Nimmo, F., and R. Pappalardo (2016), Ocean worlds in the outer solar system, Journal of Geophysical Research: Planets, 121(8), 1378-1399. 
Constraints on lateral shell thickness variations, Icarus, 191(1), 183-192.

Pappalardo, R., L. Prockter, D. Senske, R. Klima, S. Fenton Vance, and K. Craft (2016), Science objectives and capabilities of the NASA Europa Mission, in Lunar and Planetary Science Conference, vol. 47, p. 3058.

Pappalardo, R. T., J. W. Head, R. Greeley, R. J. Sullivan, C. Pilcher, G. Schubert, W. B. Moore, M. H. Carr, J. M. Moore, M. J. S. Belton, and D. L. Goldsby (1998), Geological evidence for solid-state convection in Europa's ice shell, Nature, 391 (6665), 365-368.

Rathbun, J., N. Rodriguez, and J. Spencer (2010), Galileo PPR observations of Europa: Hotspot detection limits and surface thermal properties, Icarus, 210(2), 763-769.

Schilling, N., F. M. Neubauer, and J. Saur (2007), Time-varying interaction of europa with the jovian magnetosphere: Constraints on the conductivity of europa's subsurface ocean, Icarus, 192(1), 41-55.

Schulze-Makuch, D., and L. N. Irwin (2002), Energy cycling and hypothetical organisms in Europa's ocean, Astrobiology, 2(1), 105-121.

Shrinivas, A., and G. Hunt (2014), Unconfined turbulent entrainment across density interfaces, J. Fluid Mech., $757(1)$, 573-598.

Siegert, M., J. Ellis-Evans, M. Tranter, C. Mayer, J. Petit, A. Salamatin, and J. Priscu (2001), Physical, chemical and biological processes in Lake Vostok and other Antarctic subglacial lakes, Nature, 414, 603-609.

Soderlund, K., B. Schmidt, J. Wicht, and D. Blankenship (2014), Ocean-driven heating of Europa's icy shell at low latitudes, Nature Geoscience, 7(1), 16-19. 
${ }_{354}$ Spencer, J. R., L. K. Tamppari, T. Z. Martin, and L. D. Travis (1999), Temperatures on Europa from Galileo photopolarimeter-radiometer: Nighttime thermal anomalies, Science, 284(5419), 1514-1516.

${ }_{357}$ Thomson, R. E., and J. R. Delaney (2001), Evidence for a weakly stratified europan 
Zolotov, M. Y., and J. Kargel (2009), On the chemical composition of Europa's icy shell, ocean, and underlying rocks, Europa, edited by RT Pappalardo, WB McKinnon, and K. Khurana, University of Arizona Press, Tucson, AZ, pp. 431-458.

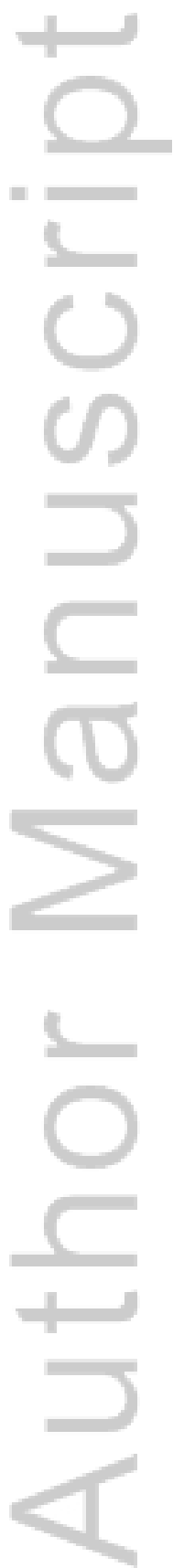

D R A F T 


\begin{tabular}{|c|c|c|c|c|}
\hline Symbol & Description & Value & Range & Unit \\
\hline$T_{s}$ & Surface temperature at the equator & 110 & - & $\mathrm{K}$ \\
\hline$T_{s}^{p \mathrm{a}}$ & Surface temperature at the pole & 52 & - & $\mathrm{K}$ \\
\hline$\kappa_{\text {ice }}$ & Thermal conductivity of ice & 2 & - & $\mathrm{W} \mathrm{m}{ }^{-1} \mathrm{~K}^{-1}$ \\
\hline & Latent heat of fusion of water & $3.3 \times 10^{8}$ & - & $\mathrm{J} \mathrm{m}^{-3}$ \\
\hline$h_{0}$ & Equilibrium ice thickness at the equator & 10 & - & $\mathrm{km}$ \\
\hline & Density of pure water & 1000 & - & $\mathrm{kg} \mathrm{m}^{-3}$ \\
\hline$\rho_{i}$ & Density of ice & 920 & - & $\mathrm{kg} \mathrm{m}{ }^{-3}$ \\
\hline$C_{P}$ & Specific heat capacity of water & 4000 & - & $\mathrm{J} \mathrm{kg}^{-1} \mathrm{~K}^{-1}$ \\
\hline$\beta(\mathrm{NaCl})^{\mathrm{b}}$ & Haline contraction coefficient of aqueous $\mathrm{NaCl}$ & $7.7 \times 10^{-4}$ & $(6.4-7.8) \times 10^{-4}$ & $\mathrm{psu}^{-1}$ \\
\hline$\beta(\mathrm{MgS}$ & Haline contraction coefficient of aqueous $\mathrm{MgSO}_{4}$ & $8.3 \times 10^{-4}$ & $(6.6-10) \times 10^{-4}$ & $\mathrm{psu}^{-1}$ \\
\hline$\alpha / \beta(\mathrm{NaCl})^{\mathrm{b}}$ & Ratio of $\alpha$ to $\beta$ for $\mathrm{NaCl}$ & 0.10 & $0-0.5$ & psu $K^{-1}$ \\
\hline$\alpha / \beta\left(\mathrm{MgSO}_{4}\right)^{\mathrm{c}}$ & Ratio of $\alpha$ to $\beta$ for $\mathrm{MgSO}_{4}$ & 0.18 & $0-0.42$ & $\operatorname{psu} K^{-1}$ \\
\hline g & Gravitational acceleration on Europa & 1.3 & - & $\mathrm{m} \mathrm{s}^{-2}$ \\
\hline$\kappa$ & Effective diffusivity & $10^{-4}$ & - & $\mathrm{m}^{2} \mathrm{~s}^{-1}$ \\
\hline$F_{b}^{\mathrm{d}}$ & Geothermal heat flux & 0.01 & $0.01-0.1$ & $\mathrm{~W} \mathrm{~m}^{-2}$ \\
\hline
\end{tabular}

a Travis et al. [2012]

b McDougall and Barker [2011]

c Vance and Brown [2013]

d Lowell and DuBose [2005]; Vance and Brown [2013]

Table 1. Freshwater layer model parameters and their approximate ranges. 
Figure 1. Model schematic depicting a low latitude (left) and high latitude (right) column. The uppermost (gray) boxes represent the ice shell. Heat is exchanged from the ocean to the ice, $F_{\text {ocn }}\left(\mathrm{W} \mathrm{m}^{-2}\right)$, and is transported away from the ocean-ice interface by diffusion. The freshwater layer is denoted in blue, with salinity $S^{e}$, temperature $T^{e}$ and depth $d$. Red lines indicate heat transport, green lines indicate salt transport, and the purple lines indicate the transport of both temperature and salinity. $F_{b}$ is the geothermal heat flux from the seafloor.

Figure 2. Salinity contrast $\Delta S$ (color-filled contours) and temperature contrast $\Delta T$ (dashed contours) between the deep ocean and the freshwater layer for seawater at an average salinity of $50 \mathrm{psu}$. For these calculations $F_{h}=1.76 \times 10^{-11} \mathrm{~m} \mathrm{~s}^{-1}$ and $F_{b}=0.01 \mathrm{~W} \mathrm{~m}^{-2}$. The black and red contours indicate $d_{\min }$ and $d_{\text {max }}$ respectively. All $\Delta T$ and $\Delta S$ values are in $\log _{10}$ space; $u^{*}$ and $d$ axes are logarithmic.

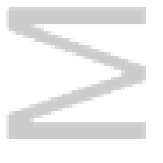

Figure 3. (a) Temperature contrast $\Delta T$ between the freshwater layer and the deep ocean corresponding to $d_{\min }$, at $u^{*}=0.01 \mathrm{~m} \mathrm{~s}^{-1}$ and $F_{h}=1.76 \times 10^{-11} \mathrm{~m} \mathrm{~s}^{-1}$, for seawater, as a function of $F_{b}$ and $S_{0}$. The black contour indicates the minimum permissible salinity. $\Delta T$ is plotted in $\log _{10}$ space. (b) Range of freshwater layer depth $d$, bounded by $d_{\min }$ and $d_{\max }$ (black and red contours respectively), temperature contrast $\Delta T$ (dashed lines) and salinity contrast $\Delta S$ (colors) as a function of $\Delta F_{\text {ocn }}\left(\mathrm{W} \mathrm{m}^{-2}\right)$, for seawater at $S_{0}=50 \mathrm{psu}, u^{*}=0.01 \mathrm{~m} \mathrm{~s}^{-1}$ and $F_{b}=0.01 \mathrm{~W} \mathrm{~m}^{-2}$. 


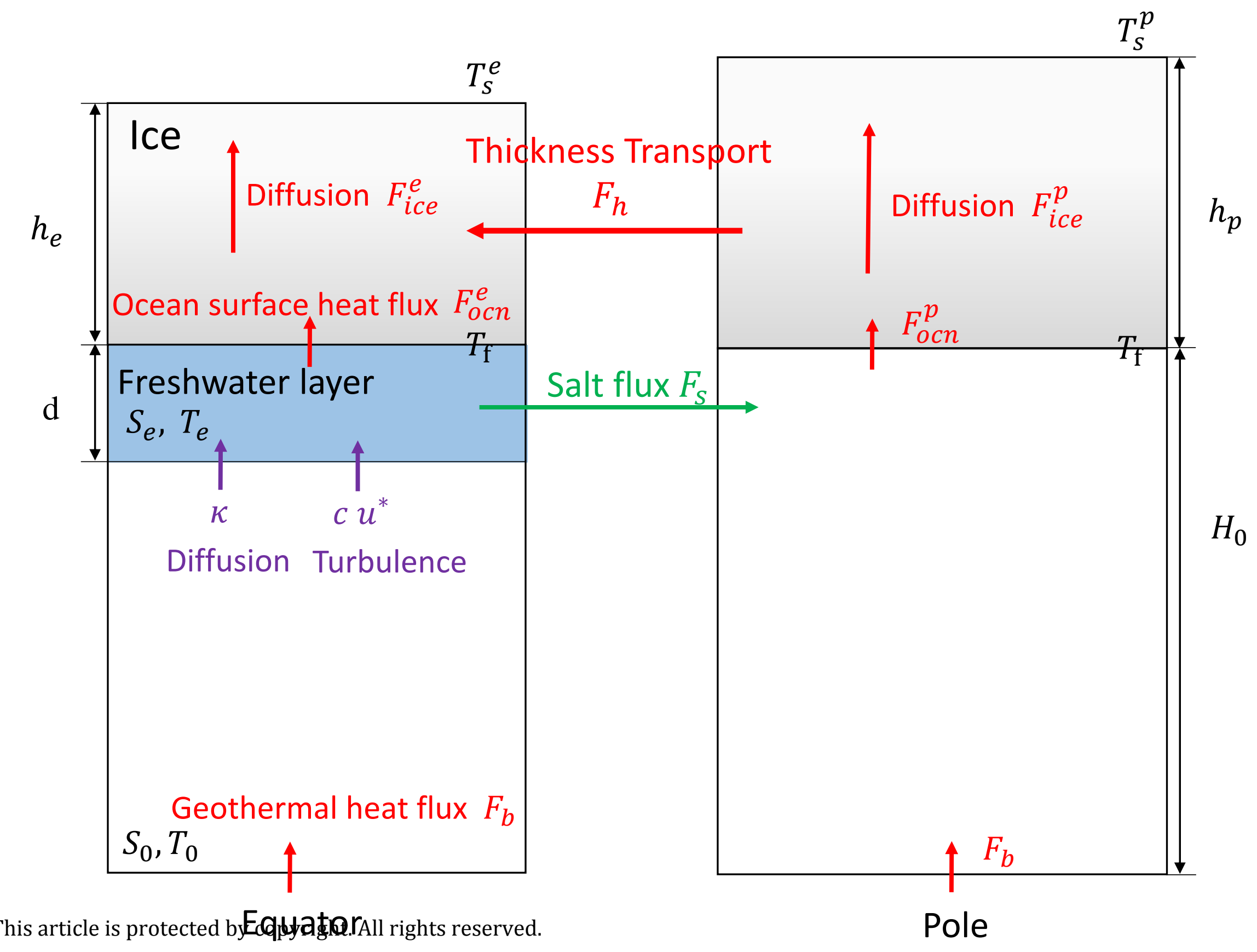




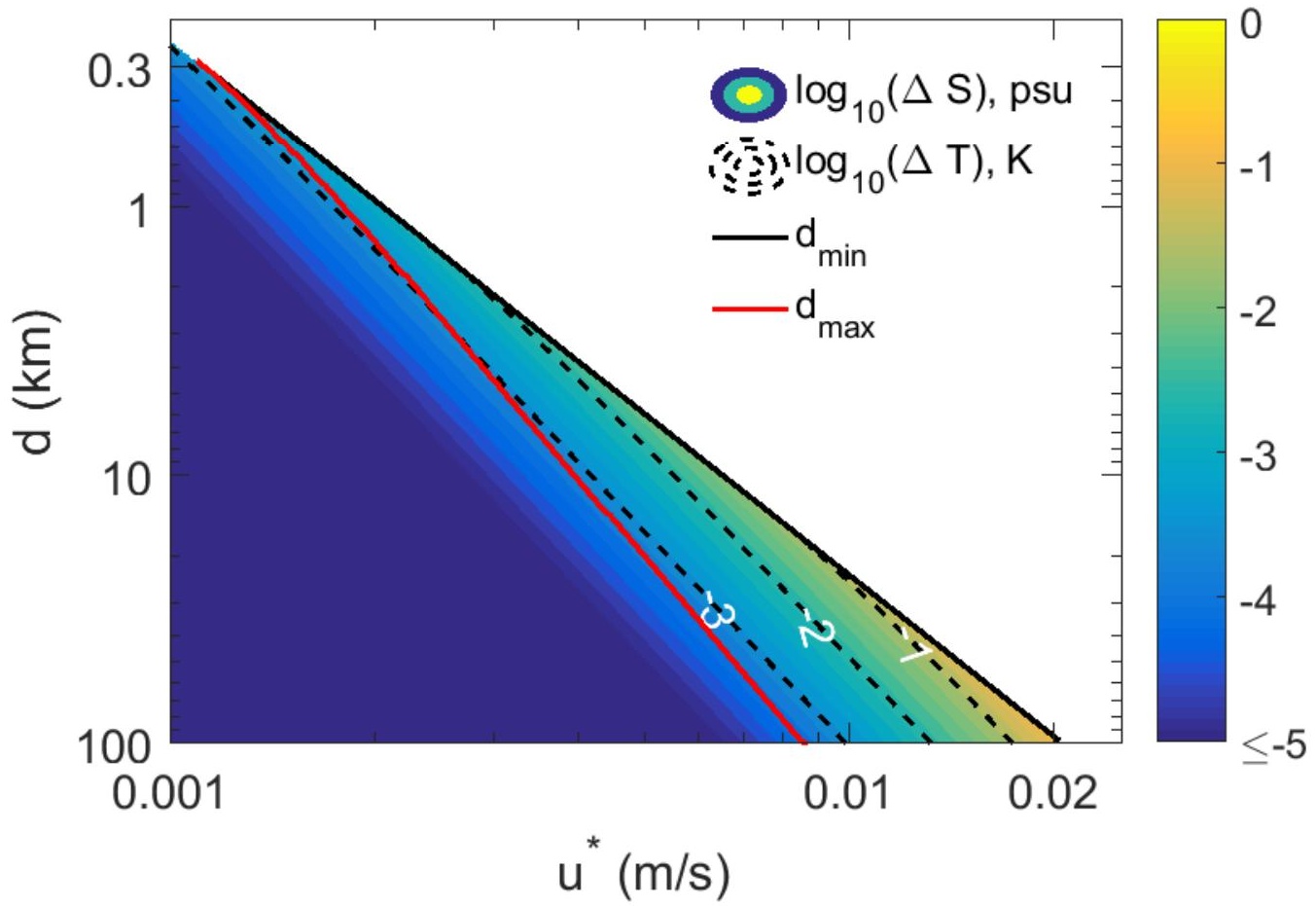



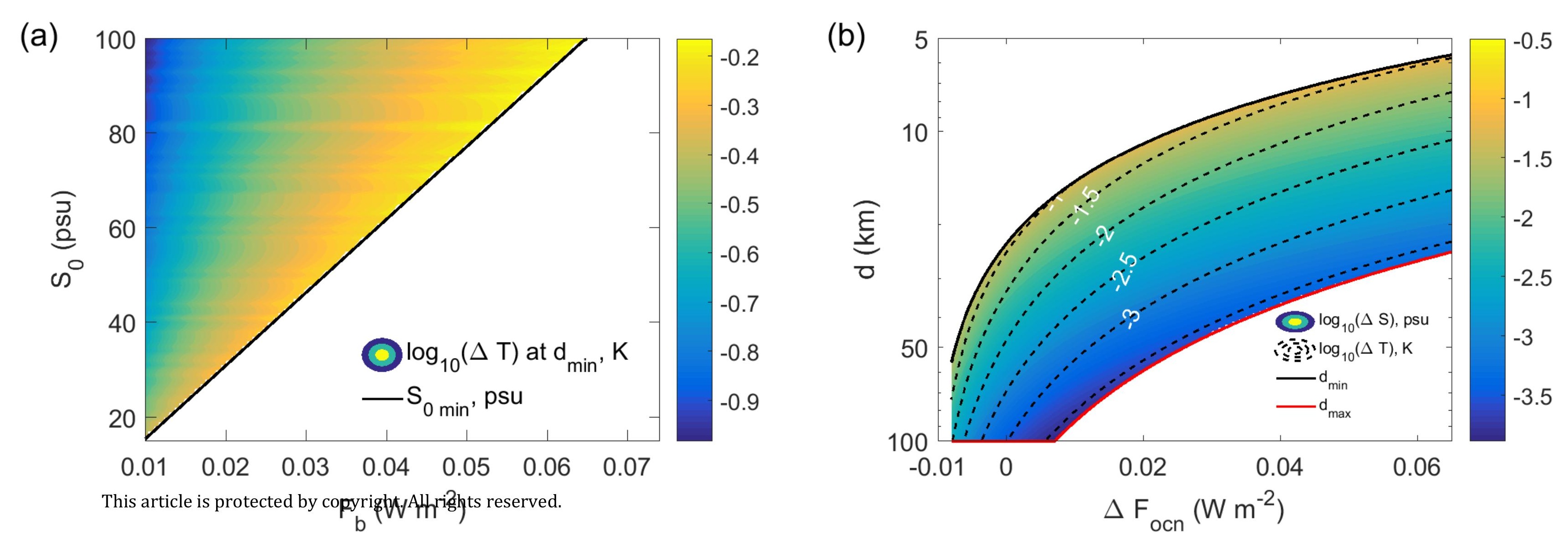